\title{
Issues of Chaos and Recurrence in Infinite Dimensions
}

\author{
Y. Charles Li \\ Dedicated to the Memory of Jerry Marsden
}

\begin{abstract}
Various issues with regard to chaos and recurrence in infinite dimensions are discussed. The doctrine we are trying to derive is that Sobolev spaces over bounded spatial domains do host chaos and recurrence, while Sobolev spaces over unbounded spatial domains are lack of chaos and recurrence. Local Sobolev spaces over unbounded spatial domains can host chaos and are natural phase spaces e.g. for fluid problems, but are very challenging to study.
\end{abstract}

\section{Contents}

1. Introduction 69

2. Lack of Recurrence for Unbounded Domain Problem 71

3. Recurrence for Bounded Spatial Domain Problem 72

4. Local Sobolev Phase Spaces $\quad 73$

5. Temporal v.s. Spatial Evolutions 74

6. Tubular Chaos $\quad 75$

7. Ubiquity of Fluid Instability $\quad 75$

$\begin{array}{ll}\text { References } & 76\end{array}$

\section{Introduction}

The first time I knew Jerry Marsden's work was through the article [2] coauthored with Phil Holmes. Early in the 1960s, Melnikov derived a simple integral measuring the splitting of stable and unstable manifolds in 2D near-integrable Hamiltonian systems. Holmes and Marsden realized the "potential" of this simple integral in proving the existence of chaos for specific differential equations. The simple Melnikov integral is actually the key! From the beginning, Holmes and Marsden eyed partial differential equations $[\mathbf{3}]$ and possible link to turbulence. They studied a beam equation following a natural approach by reducing it to pieces of ordinary

1991 Mathematics Subject Classification. Primary 35, 37; Secondary 76.

Key words and phrases. Chaos, recurrence, fluids, soliton equation, Hamiltonian system.

(C)2011 International Press 
differential equations. About 10 years later, I was working on my Ph.D. thesis at Princeton, we aimed at near-integrable soliton equations, which can be viewed as near-integrable Hamiltonian partial differential equations. This became the topic of my thesis [6], and my main research until now [10].

Simple yet important results are undoubtly the most beautiful mathematical works. Quoting from an Arnold's writing, Landau once said, along the line, that the most beautiful scientific proof should use mathematical tools not above calculus. In nowaday's mathematical society, there is a trend that a small group of mathematicians only focus on improving technicalities in other mathematician's papers. There is also a small group of mathematical journals only focusing on technicalities and problem solving. What happens to creation and innovation? While they are too difficult! Someone once made a simile: "some mathematicians are like dungbeetles competing who can dig a little deeper." This trend is poisonous to young mathematicians.

Jerry Marsden is one of the few mathematicians who do mathematical research until their last days. He is one of the "true mathematicians". Such a valuable mathematician is very rare in the global contemporary mathematical community, yet his value is so precious for the future of mathematics! In nowaday's mathematical society, there is too much political nonsense carried out by mathematicians who quitted research long ago.

Well, enough for academic and political talk, the materials below are about chaos in infinite dimensional dynamical systems which is probably Jerry's true scientific love.

Many systems in applications are infinite dimensional systems. Infinite dimensional systems are still not well studied or understood. Infinite dimensional dynamical systems have many novel features in contrast to finite dimensional dynamical systems, for example, different norms, and boundary conditions. Boundary conditions turn out to be very crucial to the dynamics, in particular whether or not the dynamics can be chaotic. For instance, decaying boundary conditions over an unbounded spatial domain severely limit the development of chaotic dynamics. The commonly observed chaotic dynamics in nature, e.g. in fluid flows, does not obey any decaying boundary condition at infinity.

There are many ways to describe chaos. Here we utilize the description by its two phenotypes: sensitive dependence on initial data and recurrence. Sensitive dependence on initial data means that no matter how small the initial condition changes, after sufficiently long time, the change will reach order one. This phenotype can also be observed in non-chaotic systems, for example, in an explosive system. Together with the second phenotype, it can often identify chaos. Recurrence means that the orbit repeatedly re-visits the neighborhood of its initial point.

Utilizing the phenotypes, one can often judge which dynamical system can host chaos and which cannot. In infinite dimensions, the dynamical systems are often defined by the Cauchy problems of partial differential equations. Here two key components influence the dynamics dramatically: the boundary condition and the norm of the phase space. As shown later on, periodic boundary condition (or other boundary conditions posed on a finite spatial domain) can often foster chaos, while decaying boundary condition (resulting in a Sobolev space on an infinite spatial domain) can hardly support chaos. For infinite spatial domain problems, relaxing 
the norm, say from a Sobolev space to a local Sobolev space, can bring chaos back into the larger phase space. But now the phase space is much more difficult to deal with.

\section{Lack of Recurrence for Unbounded Domain Problem}

Here we take the nonlinear Schrödinger equation (NLS) as the example, of course, the illustration is true for many other nonlinear wave equations.

$$
i q_{t}=q_{x x}+2|q|^{2} q,
$$

where $q$ is a complex-valued function of two real variables $(t, x)$. The NLS can be written in the Hamiltonian form

$$
-i q_{t}=\frac{\delta H}{\delta \bar{q}}
$$

where

$$
H=\int_{\mathcal{D}}\left[\left|q_{x}\right|^{2}-|q|^{4}\right] d x
$$

and $\mathcal{D}$ is either $\mathbb{T}^{1}$ or $\mathbb{R}^{1}$, in either case, the NLS is globally well-posed in the Sobolev space $H^{1}(\mathcal{D})$.

For the unbounded domain $\mathbb{R}^{1}$, the natural phase space is for example a Sobolev space $H^{1}\left(\mathbb{R}^{1}\right)$. Now we have a Hamiltonian flow defined by the Cauchy problem of NLS in the phase space $H^{1}\left(\mathbb{R}^{1}\right)$. The natural question is: Is there recurrence? Let us look at a traveling wave solution (say a soliton),

$$
q=Q(x-c t) \quad c \text { is a constant. }
$$

It is clear that the Sobolev norm $\|Q\|_{H^{s}\left(\mathbb{R}^{1}\right)}(s \geq 0$ integer) of the traveling wave solution is independent of time $t$. Thus the traveling wave solution travels on the surface of a sphere $S_{*}$ in $H^{s}\left(\mathbb{R}^{1}\right)$. It is also clear that as the time $t$ approaches infinity, the traveling wave solution $Q(x-c t)$ has no limit in the phase space $H^{1}\left(\mathbb{R}^{1}\right)$. That is, the traveling wave solution $Q(x-c t)$ travels on the surface of the sphere $S_{*}$ one way and never returns - no recurrence. On the other hand, in finite dimensional Hamiltonian systems, orbits on a sphere are always recurrent.

Of course, there is a sequence of reasons to explain the new phenomenon in infinite dimensions. First, the finite dimensional invariant volume measure has no limit in infinite dimensions, therefore, the finite dimensional recurrence argument of Poincaré is not valid in infinite dimensions [15]. Second, the Sobolev sphere $S_{*}$ in $H^{s}\left(\mathbb{R}^{1}\right)$ is not compact in $H^{s}\left(\mathbb{R}^{1}\right)$, so that the compactness argument for recurrence fails [14]. Third, the Sobolev sphere $S_{*}$ in $H^{s}\left(\mathbb{R}^{1}\right)(s>0$ integer $)$ is not compactly embedded in $H^{0}\left(\mathbb{R}^{1}\right)$ since $\mathbb{R}^{1}$ is an unbounded spatial domain where the Rellich embedding fails, so that the argument of [14] for recurrence in $H^{0}\left(\mathbb{R}^{1}\right)$ fails.

In fact, general solutions of the NLS in $H^{1}\left(\mathbb{R}^{1}\right)$ are asymptotic to multiple soliton solutions of the form

$$
q=Q\left(x-c_{1} t, \cdots, x-c_{n} t\right) \quad c_{j} \text { is a constant for } j=1, \cdots, n ;
$$

which also travel one way and never returns. Due to the lack of recurrence, one should not expect chaos in $H^{1}\left(\mathbb{R}^{1}\right)$ when the NLS is under perturbations. This claim should be true for general nonlinear wave equations in $H^{s}\left(\mathbb{R}^{n}\right)$. 


\section{Recurrence for Bounded Spatial Domain Problem}

Now we turn to the bounded spatial domain problem, e.g. the periodic domain $\mathbb{T}^{1}$. We will show that the NLS flow is recurrent in $H^{1}\left(\mathbb{T}^{1}\right)$.

Theorem 3.1. For any $q \in H^{1}\left(\mathbb{T}^{1}\right)$, any $\delta>0$, and any $T>0$; there is a $q_{*} \in H^{1}\left(\mathbb{T}^{1}\right)$ such that

$$
F^{n_{j} T}(q) \in B_{\delta}^{0}\left(q_{*}\right)=\left\{q_{1} \in H^{1}\left(\mathbb{T}^{1}\right) \mid\left\|q_{1}-q_{*}\right\|_{L^{2}\left(\mathbb{T}^{1}\right)}<\delta\right\}
$$

where $\left\{n_{j}\right\}$ is an infinite sequence of positive integers, and $F^{t}$ is the evolution operator of the NLS.

Before proving the theorem, we like to remark that the theorem roughly says that any $H^{1}\left(\mathbb{T}^{1}\right)$ solution to the NLS returns repeatedly to an arbitrarily small $L^{2}\left(\mathbb{T}^{1}\right)$ neighborhood.

Proof. In order for the proof in [14] to go through, we need to show that the $H^{1}\left(\mathbb{T}^{1}\right)$ norm is controlled by the two invariants of NLS, the Hamiltonian $H$ and the $L^{2}\left(\mathbb{T}^{1}\right)$ norm. Then any solution in $H^{1}\left(\mathbb{T}^{1}\right)$ shall stay in a bounded region $B$ in $H^{1}\left(\mathbb{T}^{1}\right)$ for all time. By the Rellich embedding theorem, $B$ is compactly embedded in $L^{2}\left(\mathbb{T}^{1}\right)$. Thus one can expect recurrence of the solution in $L^{2}\left(\mathbb{T}^{1}\right)$ as shown in [14]. By the Gagliardo-Nirenberg interpolation inequality [18] [19],

$$
\|q\|_{L^{4}} \leq C_{1}\left\|q_{x}\right\|_{L^{2}}^{1 / 4}\|q\|_{L^{2}}^{3 / 4}+C_{2}\|q\|_{L^{2}} .
$$

Using Young's inequality, one gets

$$
\begin{aligned}
\|q\|_{L^{4}}^{4} & \leq C\left(\left\|q_{x}\right\|_{L^{2}}\|q\|_{L^{2}}^{3}+\|q\|_{L^{2}}^{4}\right) \\
& \leq C\left(\kappa\left\|q_{x}\right\|_{L^{2}}^{2}+\frac{1}{\kappa}\|q\|_{L^{2}}^{6}+\|q\|_{L^{2}}^{4}\right) \\
& =\frac{1}{2}\left\|q_{x}\right\|_{L^{2}}^{2}+2 C^{2}\|q\|_{L^{2}}^{6}+C\|q\|_{L^{2}}^{4},
\end{aligned}
$$

by choosing $\kappa=\frac{1}{2 C}$. Thus,

$$
\|q\|_{H^{1}}^{2} \leq 2 H+\|q\|_{L^{2}}^{2}+4 C^{2}\|q\|_{L^{2}}^{6}+2 C\|q\|_{L^{2}}^{4} .
$$

This completes the proof.

For the unbounded domain $\mathbb{R}^{1}$, the $H^{1}\left(\mathbb{R}^{1}\right)$ norm of $q$ is still controlled by the two invariants of NLS, the Hamiltonian $H$ and the $L^{2}\left(\mathbb{R}^{1}\right)$ norm. In this case, $C_{2}=0$ in (3.1). Unfortunately, as mentioned before, unlike the periodic domain $\mathbb{T}^{1}$ case, $H^{1}\left(\mathbb{R}^{1}\right)$ is not compactly embedded in $L^{2}\left(\mathbb{R}^{1}\right)$ due to the failure of Rellich's embedding theorem; and the compactness argument in [14] cannot be carried through.

Next, we want to discuss the NLS under a Hamiltonian perturbation

$$
-i q_{t}=\frac{\delta \hat{H}}{\delta \bar{q}}, \quad \hat{H}=H+H_{1}
$$

where $H_{1}$ is the perturbation. The same argument as in Theorem 3.1 and in [14] implies the following theorem.

Theorem 3.2. If (a). the $L^{2}\left(\mathbb{T}^{1}\right)$ norm of $q$ is still an invariant for the perturbed NLS, (b). the $H^{1}\left(\mathbb{T}^{1}\right)$ norm of $q$ is controlled by $\hat{H}$ and $\|q\|_{L^{2}\left(\mathbb{T}^{1}\right)}$; then the recurrence theorem 3.1 holds for the perturbed NLS.

A simple condition that guarantees the invariance of $\|q\|_{L^{2}\left(\mathbb{T}^{1}\right)}$ is: $\bar{q} \frac{\delta H_{1}}{\delta \bar{q}}$ is real. 


\section{Local Sobolev Phase Spaces}

Sobolev space $H^{s}\left(\mathbb{R}^{n}\right)$ seems not able to host chaos. The decaying boundary condition at infinity limits the development of chaos. The natural next candidate to study is the local Sobolev space $H_{l o c}^{s}\left(\mathbb{R}^{n}\right)$. Since it contains the subspace $H^{s}\left(\mathbb{T}^{n}\right)$, $H_{l o c}^{s}\left(\mathbb{R}^{n}\right)$ can certainly host chaos. On the other hand, $H_{l o c}^{s}\left(\mathbb{R}^{n}\right)$ is a large space that is difficult to study. Usually there is no invariant manifold structure in it. One can often expects an invariant manifold structure in a Banach space with a countable base in which case the flow is equivalent to a system of infinite ordinary differential equations. Here $H_{l o c}^{s}\left(\mathbb{R}^{n}\right)$ does not have a countable base. In fact, often the unstable, center and stable subspaces in $H_{l o c}^{s}\left(\mathbb{R}^{n}\right)$ are not separated from each other [12]. Even though it is challenging to study, $H_{l o c}^{s}\left(\mathbb{R}^{n}\right)$ is often the natural phase space in applications, for example, in fluid dynamics. Take the plane Couette flow as the specific example. The plane Couette flow is governed by the NavierStokes equations

$$
\partial_{t} u_{i}+u_{j} u_{i, j}=-p_{, i}+\epsilon u_{i, j j}, \quad u_{i, i}=0
$$

where $\left(u_{1}, u_{2}, u_{3}\right)$ are the three components of the fluid velocity along $(x, y, z)$ directions, $p$ is the pressure, and $\epsilon=1 / R$ is the inverse of the Reynolds number. The boundary condition is

$$
u_{1}(x, a, z)=\alpha, \quad u_{1}(x, b, z)=\beta, \quad u_{j}(x, a, z)=u_{j}(x, b, z)=0,(j=2,3) ;
$$

where $a<b, \alpha<\beta$, and $u_{i}(i=1,2,3)$ are bounded in $x$ and $z$. In this case, the natural phase space (manifold) is

$$
H_{l o c}^{s}\left(\mathbb{R}^{1} \times[a, b] \times \mathbb{R}^{1}\right)
$$

under the constraint of the boundary condition (4.2). Due to the difficulty in studying this phase space, current studies (both numerical and analytical) focus upon the restriction that $u$ is periodic in $x$ and $z$ in which case the spatial domain is bounded and the phase space (manifold) is simplified to

$$
H^{s}\left(\mathbb{T}^{1} \times[a, b] \times \mathbb{T}^{1}\right)
$$

under the constraint of the boundary condition (4.2).

An interesting subspace of $H_{l o c}^{s}\left(\mathbb{R}^{1}\right)$ is the space of spatially quasi-periodic functions:

$$
H_{\text {quasi }}^{s}\left(\mathbb{R}^{1}\right)=\left\{q \in H_{\text {loc }}^{s}\left(\mathbb{R}^{1}\right) \mid q(x)=Q\left(\omega_{1} x, \omega_{2} x, \cdots, \omega_{n} x\right)\right\}
$$

where $\left(\omega_{1}, \omega_{2}, \cdots, \omega_{n}\right)$ is a quasi-periodic base. Even though it still does not have a countable base, the special nature of $H_{\text {quasi }}^{s}\left(\mathbb{R}^{1}\right)$ may make it easier to study. Again take the NLS (2.1) as the example, one can obtain an explicit expression for a homoclinic orbit which is spatially quasi-periodic [12]:

$$
q(t, x)=\tilde{Q}+q_{0}(t) \sin \hat{\vartheta}_{0} \Pi_{2} / \Pi_{1}
$$

where

$$
q_{0}(t)=a e^{i \theta(t)}, \quad \theta(t)=-\left[2 a^{2} t+\gamma\right],
$$


and $a$ is the amplitude and $\gamma$ is the phase;

$$
\begin{aligned}
\tilde{Q}= & q_{0}(t)\left[1+\sin \vartheta_{0} \operatorname{sech} \tau \cos X\right]^{-1}\left[\cos 2 \vartheta_{0}\right. \\
& \left.-i \sin 2 \vartheta_{0} \tanh \tau-\sin \vartheta_{0} \operatorname{sech} \tau \cos X\right] \\
\Pi_{1}= & {\left[\left(\sin \hat{\vartheta}_{0}\right)^{2}\left(1+\sin \vartheta_{0} \operatorname{sech} \tau \cos X\right)^{2}+\frac{1}{8}\left(\sin 2 \vartheta_{0}\right)^{2}(\operatorname{sech} \tau)^{2}(1-\cos 2 X)\right] } \\
& \left(1+\sin \hat{\vartheta}_{0} \operatorname{sech} \hat{\tau} \cos \hat{X}\right)-\frac{1}{2} \sin 2 \vartheta_{0} \sin 2 \hat{\vartheta}_{0} \operatorname{sech} \tau \operatorname{sech} \hat{\tau} \\
& \left(1+\sin \vartheta_{0} \operatorname{sech} \tau \cos X\right) \sin X \sin \hat{X}+\left(\sin \vartheta_{0}\right)^{2}\left[1+2 \sin \vartheta_{0}\right. \\
& \left.\operatorname{sech} \tau \cos X+\left[(\cos X)^{2}-\left(\cos \vartheta_{0}\right)^{2}\right](\operatorname{sech} \tau)^{2}\right] \\
& \left(1+\sin \hat{\vartheta}_{0} \operatorname{sech} \hat{\tau} \cos \hat{X}\right)-2 \sin \hat{\vartheta}_{0} \sin \vartheta_{0}\left[\cos \hat{\vartheta}_{0} \cos \vartheta_{0}\right. \\
& \left.\tanh \hat{\tau} \tanh \tau+\left(\sin \vartheta_{0}+\operatorname{sech} \tau \cos X\right)\left(\sin \hat{\vartheta}_{0}+\operatorname{sech} \hat{\tau} \cos \hat{X}\right)\right] \\
& \left(1+\sin \vartheta_{0} \operatorname{sech} \tau \cos X\right), \\
\Pi_{2}= & {\left[-2\left(\sin \hat{\vartheta}_{0}\right)^{2}\left(1+\sin \vartheta_{0} \operatorname{sech} \tau \cos X\right)^{2}+\frac{1}{4}\left(\sin 2 \vartheta_{0}\right)^{2}(\operatorname{sech} \tau)^{2}\right.} \\
& (1-\cos 2 X)]\left(\sin \hat{\vartheta}_{0}+\operatorname{sech} \hat{\tau} \cos \hat{X}+i \cos \hat{\vartheta}_{0} \tanh \hat{\tau}\right) \\
& +2\left(\sin \vartheta_{0}\right)^{2}\left(-\cos \vartheta_{0} \tanh \tau+i \sin \vartheta_{0}+i \operatorname{sech} \tau \cos X\right)^{2} \\
& \left(\sin \hat{\vartheta}_{0}+\operatorname{sech} \hat{\tau} \cos \hat{X}-i \cos \hat{\vartheta}_{0} \tanh \hat{\tau}\right)+2 \sin \vartheta_{0}\left(\sin \vartheta_{0}\right. \\
& \left.+\operatorname{sech} \tau \cos X+i \cos \vartheta_{0} \tanh \tau\right)\left[2 \sin \hat{\vartheta}_{0}\left(1+\sin \vartheta_{0} \operatorname{sech} \tau \cos X\right)\right. \\
& \left.\left(1+\sin \hat{\vartheta}_{0} \operatorname{sech} \hat{\tau} \cos \hat{X}\right)-\sin 2 \vartheta_{0} \cos \hat{\vartheta}_{0} \operatorname{sech} \tau \operatorname{sech} \hat{\tau} \sin X \sin \hat{X}\right] \\
&
\end{aligned}
$$

and

$$
\begin{aligned}
& \beta_{1}+i \sqrt{a^{2}-\beta_{1}^{2}}=a e^{i \vartheta_{0}}, \quad \beta_{2}+i \sqrt{a^{2}-\beta_{2}^{2}}=a e^{i \hat{\vartheta}_{0}}, \\
& \tau=4 \sqrt{a^{2}-\beta_{1}^{2}} \beta_{1} t-\rho, \quad \hat{\tau}=4 \sqrt{a^{2}-\beta_{2}^{2}} \beta_{2} t-\hat{\rho}, \\
& X=2 \beta_{1} x+\vartheta-\vartheta_{0}+\pi / 2, \quad \hat{X}=2 \beta_{2} x+\hat{\vartheta}-\hat{\vartheta}_{0}+\pi / 2 ;
\end{aligned}
$$

and $0<\beta_{1}, \beta_{2}<a, a, \gamma, \rho, \hat{\rho}, \vartheta$, and $\hat{\vartheta}$ are real parameters. As $t \rightarrow \pm \infty$,

$$
q(t, x) \rightarrow q_{0}(t) e^{\mp i 2\left(\vartheta_{0}+\hat{\vartheta}_{0}\right)},
$$

that is, $q(t, x)$ is homoclinic to the uniform periodic orbit $q_{0}(t)$ (4.4) up to phase translations. The unstable, center and stable subspaces in $H_{\text {quasi }}^{s}\left(\mathbb{R}^{1}\right)$ of the uniform periodic orbit $q_{0}(t)$ (4.4) are not separated from each other [12]. The interesting question here is: Does the homoclinic orbit (4.3) induce chaos when the NLS is under perturbations?

\section{Temporal v.s. Spatial Evolutions}

From a dynamical system point of view, a natural evolution in infinite dimensions is a temporal one posed by the Cauchy problem of a partial differential 
equation. For such a Cauchy problem, looking at its spatial evolution is often awkward and ill-posed. Take the simple example: $u_{t}=u_{x x}, x \in \mathbb{R}$. For temporal evolution, one may choose the phase space to be the local space $H_{l o c}^{3}\left(\mathbb{R}^{1}\right)$. A simple orbit in $H_{\text {loc }}^{3}\left(\mathbb{R}^{1}\right)$ is

$$
u=u_{0} e^{-t k^{2}}, u_{0}=\cos k x, k \in \mathbb{R}^{1} .
$$

If this were a nonlinear equation, one could ask the question of sensitive dependence of $u$ on the initial data $u_{0}$, and the existence of temporal chaos. For all the orbits in $H_{\text {loc }}^{3}\left(\mathbb{R}^{1}\right.$ ), one can also look at their spatial evolution (development may be a better word). For example, one can rewrite the simple orbit as

$$
u=u^{0} \cos k x, u^{0}=e^{-t k^{2}}, k \in \mathbb{R}^{1} .
$$

One can equip a temporal topology to these orbits, e.g. $C^{0}\left(\mathbb{R}^{+}\right)$topology: $\|u\|_{C^{0}}=$ $\sup _{t \in \mathbb{R}^{+}}|u(t)|$. Obviously

$$
u^{0}=e^{-t k^{2}} \in C^{0}\left(\mathbb{R}^{+}\right) \text {, and } u \in C^{0}\left(\mathbb{R}^{+}\right) \text {for any } x \in \mathbb{R} .
$$

If this were a nonlinear equation, one could ask the question of sensitive dependence of $u$ on the initial data $u^{0}$ under the topology $C^{0}\left(\mathbb{R}^{+}\right)$, and the existence of spatial chaos.

\section{Tubular Chaos}

The two phenotypes of chaos can often be realized near a homoclinic orbit (or a heteroclinic cycle). When the stable and unstable manifolds of a saddle intersect transversally and form a tangle with a homoclinic orbit, recurrence can occur near the homoclinic orbit. The Lyapunov exponents near the homoclinic orbit will be positive due to the transversality of the intersection between the stable and unstable manifolds along the homoclinic orbit. The positive Lyapunov exponents lead to sensitive dependence upon initial data.

In higher dimensions, it is natural to study homoclinic tubes (heteroclinically tubular cycles) instead of homoclinic orbits (heteroclinic cycles). It turns out that like homoclinic orbits (heteroclinic cycles), homoclinic tubes (heteroclinically tubular cycles) can also lead to chaos - tubular chaos $[\mathbf{7}][\mathbf{8}][\mathbf{9}][\mathbf{1 3}]$. Such a tubular chaos is represented by a Bernoulli shift dynamics on a Cantor set of submanifolds instead of points. These submanifolds can still contain finer scale chaos inside them, and continuing this process can lead to a chain of finer and finer scale chaos and form a chaos cascade [9]. The existence of tubular chaos shows that taking the averages of solutions with respect to a neighborhood of initial data will not eliminate the chaotic nature of the dynamics.

\section{Ubiquity of Fluid Instability}

One common feature of fluid flows is that it is easy for them to become unstable. Rarely there is a fluid flow that is stable for all values of its parameters. So there must be something universal (generic) that makes fluid flows unstable. The spatial oscillation $e^{i k \cdot x}$ could be the thing. Since $e^{i k \cdot x}$ is a Fourier mode, it is certainly universal. Often the larger the $k$ is, the more unstable modes the oscillation $e^{i k \cdot x}$ induces. This will lead to more spatial disorder - more turbulent states. Next we mention some examples.

For the 2D Kolmogorov flow (with a periodic boundary condition in each direction and an artificial force), spatially oscillatory shears $(\cos k y, 0)$ are steady states. 
It turns out that the larger the $k$ is, the more unstable modes the shear has [5] [12]. For the 2D plane Couette flow, even though the linear shear $(y, 0)$ is linearly stable for all values of the Reynolds number [20], adding "small" spatial oscillations $\left(y+\frac{A}{n} \sin (4 n \pi y), 0\right)$ will end up linearly unstable shears (they are steady for the inviscid flow, and slowly drifting for the viscous flow) for any $n$ and $A \in\left(\frac{1}{8 \pi}, \frac{1}{4 \pi}\right)$ [16]. The larger the $n$ is, the more unstable modes it has.

Intuitively it is very easy to understand the destablizing effect of the smallamplitude high-frequency spatial oscillations. By the well-known Rayleigh criterion, a necessary condition for a shear $U(y)$ to have inviscid linear instability is that it has an inflection point. If a shear $U(y)$ does not have any inflection point, adding the small-amplitude high-frquency spatial oscillations can create inflection points even though such oscillations do not change the original velocity profile much. That is, the slight modification on the velocity profile $U(y)$,

$$
\tilde{U}(y)=U(y)+\frac{A}{n} \sin (n y) \text { for large } n
$$

can have a significant modification on its second derivative $U^{\prime \prime}(y)$,

$$
\tilde{U}^{\prime \prime}(y)=U^{\prime \prime}(y)-A n \sin (n y) \text { for large } n
$$

and creates a lot of inflection points and the potential of linear instabilities. Both shears $U(y)$ and $\tilde{U}(y)$ are steady states under the Euler dynamics. Under the Navier-Stokes dynamics, they may not be steady rather drift slowly in time. Such slowly drifting states can still play an important role in transient turbulence,

In some sense, spatial oscillations are recurrent motions - spatial shakings. Even small amplitude but high frequency spatial oscillations can generate considerable vorticities. Derivatives of vorticities are even higher. Such structures not only make the fluid flows unstable, but also create a lot of unstable modes leading to more turbulent spatial disorders. Temporally oscillatory forcings are also known to generate instabilities of fluid flows, for example the well-known Faraday wave generation $[\mathbf{1}][\mathbf{4}][\mathbf{2 1}]$.

\section{References}

[1] M. Faraday, On a peculiar class of acoustical figures, and on certain forms assumed by groups of particles upon vibrating elastic surfaces, Phil. Trans. R. Soc. Lond. 121 (1831), 299-340.

[2] P. Holmes, J. Marsden, Horseshoes in perturbations of Hamiltonian systems with two degrees of freedom, Comm. Math. Phys. 82, no.4 (1981/82), 523544.

[3] P. Holmes, J. Marsden, A partial differential equation with infinitely many periodic orbits: chaotic oscillations of a forced beam, Arch. Rational Mech. Anal. 76, no.2 (1981), 135165.

[4] A. Kudrolli, J. Gollub, Localized spatiotemporal chaos in surface waves, Phys. Rev. E. 54, no.2 (1996), 1052-1055.

[5] Y. Latushkin, Y. Li, M. Stanislavova, The Spectrum of a linearized 2D Euler operator, Studies in Applied Mathematics 112 (2004), 259-270.

[6] Y. Li, Chaotic Dynamics in Partial Differential Equations and Lattices, Princeton University, Ph.D. Dissertation, (1993).

[7] Y. Li, Chaos and shadowing lemma for autonomous systems of infinite dimensions, J. Dyn. Diff. Eq. 15, no.4 (2003), 699-730.

[8] Y. Li, Chaos and shadowing around a homoclinic tube, Abstract and Applied Analysis 2003, no.16 (2003), 923-931.

[9] Y. Li, Homoclinic tubes and chaos in perturbed sine-Gordon equation, Chaos, Solitons and Fractals 20, no.4 (2004), 791-798.

[10] Y. Li, Chaos in Partial Differential Equations, International Press, (2004). 
[11] Y. Li, Invariant manifolds and their zero-viscosity limits, Dynamics of PDE 2, no.2 (2005), 159-186.

[12] Y. Li, On quasi-periodic boundary condition problem, J. Math. Phys. 46, no.1 (2005), 013503.

[13] Y. Li, Chaos and shadowing around a heteroclinically tubular cycle with an application to sine-Gordon equation, Studies in Applied Mathematics 116 (2006), 145-171.

[14] Y. Li, A recurrence theorem on the solutions to the 2D Euler equation, Asian J. Math 13, no.1 (2009), 1-6. Also available at: http://www.math.missouri.edu/ ${ }^{\circ}$ cli/Recurrence2.pdf

[15] Y. Li, The Poincaré recurrence problem of inviscid incompressible fluids, Asian J. Math 13, no.1 (2009), 7-14. Also available at: http://www.math.missouri.edu/ cli/Recurrence.pdf

[16] Y. Li, Z. Lin, A resolution of the Sommerfeld paradox, Submitted (2009).

[17] V. Melnikov, On the stability of the center for time periodic perturbations, Trans. Moscow Math. Soc. 12 (1963), 1-57.

[18] L. Nirenberg, On elliptic partial differential equations (Lecture II), Ann. Sc. Norm. Sup. Pisa 13 (1959), 123-131.

[19] L. Nirenberg, An extended interpolation inequality, Ann. Sc. Norm. Sup. Pisa 20 (1966), 733-737.

[20] V. Romanov, Stability of plane-parallel Couette flow, Functional Analysis and Its Applications 7, no.2 (1973), 137-146.

[21] M.-T. Westra et al., Patterns of Faraday waves, J. Fluid Mech. 496 (2003), 1-32.

Department of Mathematics, University of Missouri, Columbia, MO 65211

E-mail address: liyan@missouri.edu 